\title{
PHYSICS OF STIMULATED EMISSION IN BLUE SEMICONDUCTOR LASERS
}

\author{
A.V. NURMIKKo* \\ Division of Engineering and Department of Physics \\ Brown University, Providence RI 02912, USA
}

In this article an overview is given about the special properties of the new blue and green semiconductor lasers, with emphasis on those basic processes that power the stimulated emission in these compact devices. Of special interest are the strong electron-hole Coulomb correlations which can be spectroscopically identified as unique features in quantum wells of wide band gap semiconductors.

PACS numbers: 42.55.Px, 42.55.Sa, 71.55.Eq

\section{Introduction}

The past few years have brought to life a new class of semiconductor diode lasers, spanning the spectral range from the violet to the green, based on wide band gap semiconductors. The first laser demonstrations in the blue/green were made in ZnSe-based quantum well (QW) heterostructures [1]. This has been followed by the rapid recent developments in the GaN-compounds that have brought the latter devices to the threshold of technological viability [2]. The presentation by S. Nakamura in this Jabłoński Centennial Conference offers several examples of the progress with the light emitting nitride devices, including LEDs that span the wavelength range from near ultraviolet to amber.

In this paper we focus on the rich realm of optical physics that the wide band gap semiconductor lasers offer, specifically in terms of light-matter coupling within a high density two-dimensional system electron-hole in a $\mathrm{ZnCdSe}$ or InGaN quantum well. Although these ternary compounds that define active optical media are expected to be rather similar in terms of (effective mass theory) expectations, namely that strong Coulomb (excitonic) correlations are much more dominant that in conventional semiconductor lasers, we find considerable contrasts between the wide gap II-VI and III-V systems at present. The many-electron contributions to the physics of optical gain and stimulated emission in the nitride diode lasers are largely masked by the presence of a significant amount of spatial compositional fluctuations in the InGaN QW material. The "disorder" arises from the

*e-mail: Arto_Nurmikko@Brown.edu 
non-random nature of the ternary semiconductor, leading to partial clustering of In-rich regions whose size spans a wide distribution range from atomic to mesoscopic $(\approx 100 \mathrm{~nm})$ scale. The ZnSe-based QWs enjoy the benefit of very high quality heteroepitaxy and small crystalline disorder, as a consequence of which excitonic contributions (in the Wannier limit) can be readily observed to make a profound enhanced contributions to optical gain, with characteristic spectral fingerprints. As shown below, at cryogenic temperatures, for example, excitonic molecules dominate optical gain which can reach peak values of the gain coefficient in excess of $g>10^{5} \mathrm{~cm}^{-1}$. In the room temperature continuous-wave diode lasers, the excitonic enhancements remain very potent and aid in reducing the threshold current densities to values below $200 \mathrm{~A} / \mathrm{cm}^{2}$. The importance of pairwise Coulomb correlation of the electron-holes can be directly seen, for example, by studying the emission spectra of the diode lasers in an external magnetic field. As a final illustration of the excitonic aspect of optical gain in a $\mathrm{ZnCdSe} \mathrm{QW}$, we describe experiments in vertical cavity microresonators in which the light-matter interaction is enhanced by the so-called normal mode coupling between the exciton and photon oscillators (Rabi splitting analog in atomic systems).

\section{Excitons in wide gap semiconductor quantum wells}

The role of excitons in wide gap semiconductors has been recognized for three decades, with much experimental work obtained from linear optical studies on bulk materials at low temperatures. Only recently, however, has it been recognized that excitonic effects are also of significance at room temperature quantum well light emitters, thus creating a natural bridge between basic and applied optical device physics.

Figure 1 shows the absorption spectra for $\mathrm{ZnCdSe} / \mathrm{ZnSSe} \mathrm{QWs}[3]$ and a GaN [4] thin epitaxial layer, respectively. Direct transmission measurements yielded the data in (a), whereas the absorption spectrum was obtained from spectroscopic ellipsometry in (b). The II-VI example shows the impact of dimensionality on the exciton oscillator strength and binding energy: the sample with well thickness of $200 \AA$ is analogous to a bulk material while the sample with $L_{\mathrm{w}}=30 \AA$ is near the 2D limit (relative to bulk exciton Bohr diameter). Note how the $n=1$ QW heavy-hole exciton peak dominates the absorption well beyond room temperature, with a peak value in excess of $10^{5} \mathrm{~cm}^{-1}$. Its binding energy has been measured from diamagnetic shifts in high magnetic fields to be approximately $40 \mathrm{meV}$, hence exceeding $k T$ (at room temperature) as well as the optical phonon energy (which provides a mechanism for exciton dissociation via inelastic collisions). In case of InGaN QWs, the alloy compositional anomalies has so far prevented comparable measurements (absorption edges are simply too much broadened by the crystalline microstructure). On the other hand, even for bulk GaN film, a distinct exciton absorption peak is obtained in Fig. 1b, with an estimated binding energy of about $22 \mathrm{meV}$. Accordingly, we may anticipate that strong exciton effects are present in the InGaN QWs, even if masked to first order by the microstructural complexity.

Figure 2 shows the comparison between the absorption edge at the $n=1$ heavy hole $(\mathrm{HH})$ exciton in a $\mathrm{ZnCdSe} \mathrm{QW}$ and the corresponding electrolumines- 

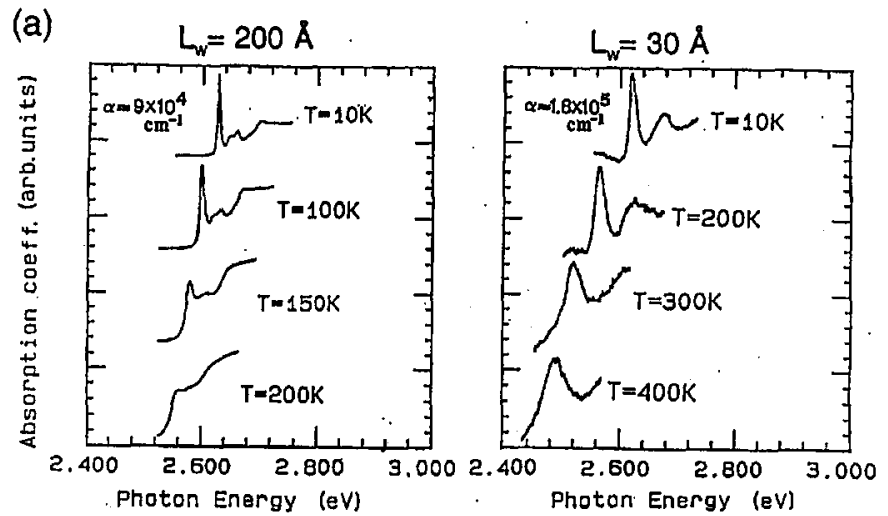

(b)

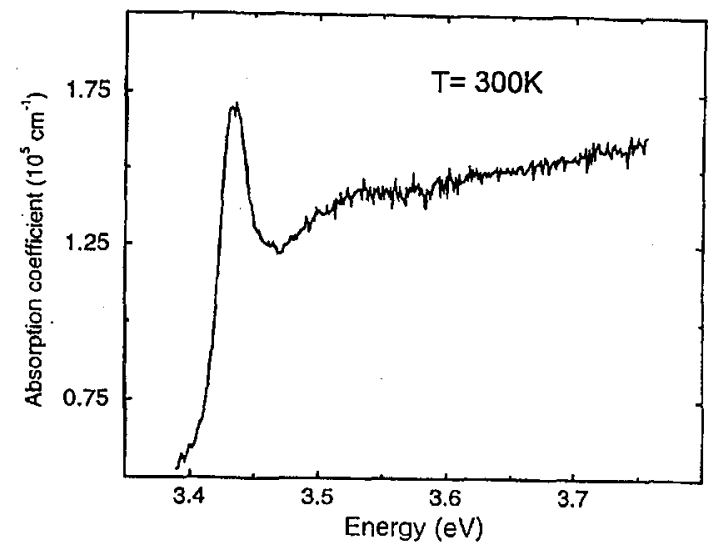

Fig. 1. Excitonic absorption in (a) $\mathrm{ZnCdSe} / \mathrm{ZnSe}$ heterostructures and (b) $\mathrm{GaN}$ epitaxial layer. The two thicknesses in (a) correspond to three- and two-dimensional limits, respectively (after Refs. [3] and [4]).
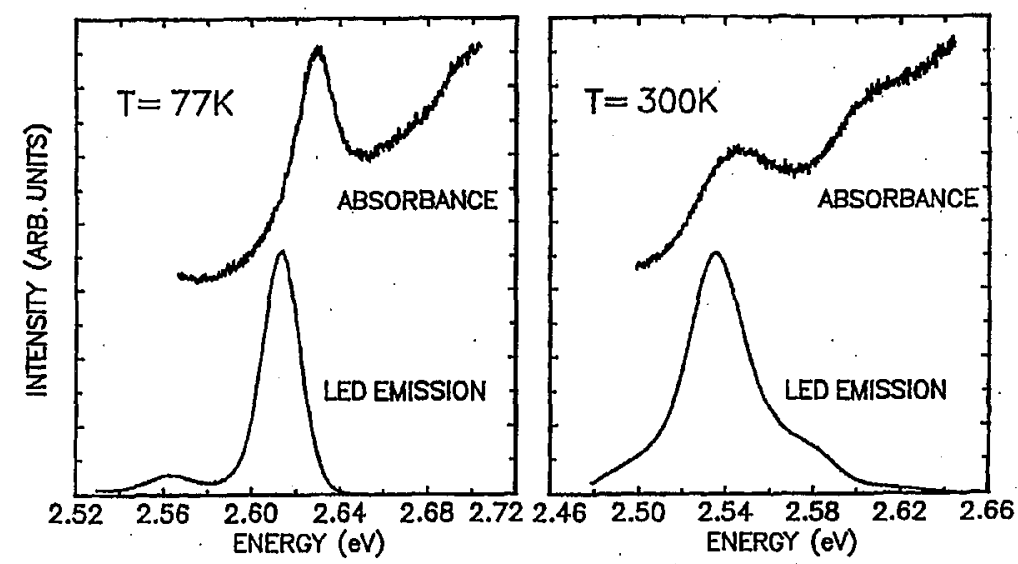

Fig. 2. Comparison between absorption and LED emission at the $n=1 \mathrm{HH}$ exciton transition in a $\mathrm{ZnCdSe} \mathrm{QW}$ diode structure (after Ref. [5]). 
cent emission from a $p n$-junction LED device [5]. Both at cryogenic and room temperature, we see a distinct spectral correlation between absorption and emission, which is indicative of the $2 \mathrm{D}$ excitonic radiative recombination in the green/blue LED. The finite redshift of the LED emission relative to the peak of the exciton absorption peak is associated with finite $\mathrm{e}-\mathrm{h}$ localization effects. These (relatively weak) localization processes originate from the random compositional fluctuations in the ternary alloy $\mathrm{Zn}_{1-x} \mathrm{Cd}_{x} \mathrm{Se}(x \approx 0.2)$. The scale of the localization due to the cation compositional anomalies increases by about one order of magnitude in the $\operatorname{In}_{x} \mathrm{Ga}_{1-x} \mathrm{~N} Q W(x \approx 0.1)$, from about 10 to $100 \mathrm{meV}$, strongly favoring radiative recombination over nonradiative processes (typically at point defects for which long carrier diffusion lengths are especially sensitive to).

Excitonic molecules are four-particle Coulomb correlated excitations which have been long known in "classic" excitonic insulators such as $\mathrm{CuCl}$ (unsuitable for electroluminescent devices). As we see in the next section, these "biexcitons" can be responsible for stimulated emission and laser action in the wide gap semiconductors. Figure 3a introduces a simplified energy level diagram, adapted from
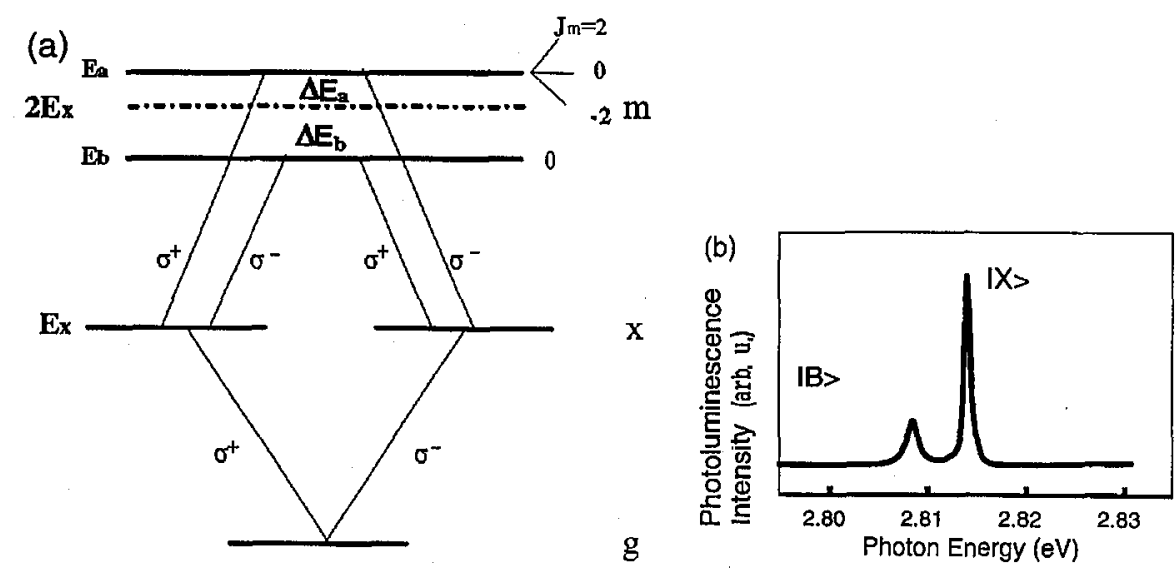

Fig. 3. (a) Schematic multilevel model diagram with relevant angular momentum and optical transition rules for the one and two-exciton system; (b) photoluminescence spectra for a single $75 \AA$ thick $\mathrm{ZnSe} Q W$ at $T=10 \mathrm{~K}$, showing the positions for the excitonic $(|X\rangle)$ and biexcitonic $(|B\rangle)$ resonances.

Ref. [6], in which the energy levels of the crystal ground state, exciton (at energy $E_{x}$ ), and the stable (bound) molecular state $\left(\right.$ at $E_{\mathrm{b}}$ ) are indicated, together with the appropriate angular momentum optical selection rules. The biexciton spectrum also includes the "antibound" state (at $E_{\mathrm{a}}$ ), which is unstable due to the repulsive nature of the Coulomb interaction in the Hartree-Fock limit (Pauli repulsion). Figure $3 \mathrm{~b}$ shows experimental photoluminescence emission under moderate excitation, acquired from a single $\mathrm{ZnSe} \mathrm{QW}$ sample at $T=10 \mathrm{~K}$, indicating the $n=1$ $\mathrm{HH}$ exciton recombination to the crystal ground state (transition labelled as $|X\rangle$ ), but also the biexciton recombination (transition labelled as $|B\rangle$ ) at a photon energy $E_{\mathrm{b}}-E_{x}$ [7]. The ZnSe QW sample is of exceptional quality so that a value 
for the molecular binding energy $\Delta E_{\mathrm{b}}=5.0 \mathrm{meV}$ is obtained with accuracy. The molecular state has been verified from a number of other optical experiments as well [6].

The "antibound biexciton states" have also been identified spectroscopically in recent experiments in ZnSe single QWs by using coherent transient nonlinear optical spectroscopy [8]. The four-wave mixing (FWM) techniques in question have been employed for a number of years to discover a wealth of information about excitons, and especially their interactions, in GaAs-based QWs. These include recent work in which the impact of many-body effects on biexciton states have been theoretically demonstrated to be of particular importance in an interacting gas of excitons [9]. The top portion of Fig. 4 shows a schematic of the typical FWM

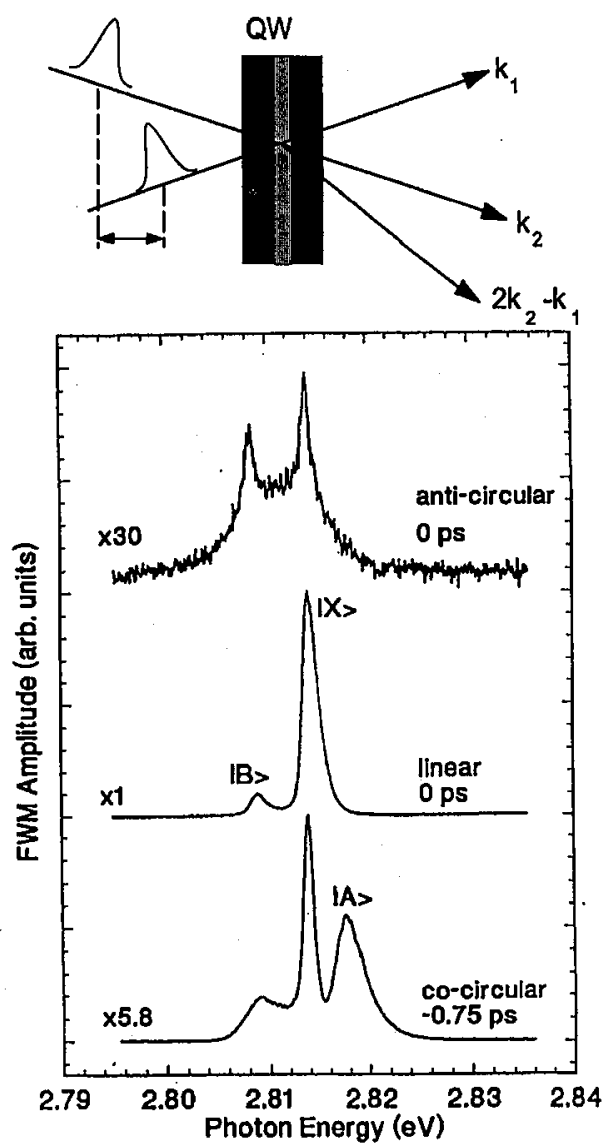

Fig. 4. Upper trace: schematic of a four-wave mixing nonlinear spectroscopic experiment. Lower panel: a comparison of the FWM spectra measured under co-circular-polarization configuration (pump-probe time delay $\tau=-0.75 \mathrm{ps}$ ) highlighting the "antibound" exciton pair transition $|A\rangle$, linear-polarization configuration $(\tau=0 \mathrm{ps})$, and anti-circular-polarization configuration $(\tau=0 \mathrm{ps})$, all under the same conditions of excitation. 
experiment in which a coherent polarization grating is created by two incident short laser pulses $\left(<10^{-13} \mathrm{~s}\right)$ with a variable time delay $\tau$. One of the beams (the "probe") is diffracted by the grating and its intensity measured as a function of $\tau$. The data in Fig. 4.shows spectrally resolved FWM signals obtained for a ZnSe QW sample at $T=10 \mathrm{~K}$. Apart from the verification of selection rules for the $|X\rangle$ and $|B\rangle$ transitions in the top trace (forbidden anticircular polarization configuration; the finite signal is due to the imperfect polarizers), the bottom trace shows the appearance of a signal at spectral position labeled as $|A\rangle$ which is only seen in the "negative time delay" regime (probe precedes the pump). This signal is interpreted as being due to the (coherent) "antibound states" and its observation for negative values of $\tau$ is consistent with theoretical expectations [9] which involve a more complete accounting and impact of the biexciton continuum states that underlie the "antibound states" in a semiconductor.

\section{Excitonic molecules and stimulated emission}

As already noted, wide gap semiconductor lasers differ both qualitatively and quantitatively from conventional GaAs-based devices in terms of the many-body physics of within the dense electron-hole gas which is necessary for the formation of optical gain for stimulated emission. In this section we focus on cryogenic temperatures where the excitonic molecules have been shown to dominate optical gain in high quality $\mathrm{ZnSe}$ and $\mathrm{ZnCdSe}$ QWs. Undoubtedly, similar and related effects will be discovered in the GaN heterostructures once their microstructure improves so as to yield the necessary spectroscopic clarity.

Experimental evidence for the biexciton gain in a $\mathrm{ZnCdSe}$ heterostructure was discovered by Henneberger and co-workers [10], as well as by Kozlov et al. in single $\mathrm{ZnSe}$ QWs [7, 11]. Figure 5 shows an example from the latter work where the top trace shows the transmission spectrum through the $50 \AA$ thick layer of the QW material with intense, narrow $(\approx 0.5 \mathrm{meV}$ ) absorption peaks at the $n=1 \mathrm{HH}$ and light hole (LH) excitons. The bottom trace shows the result of an optical pumping experiment at $T=10 \mathrm{~K}$ in which the excitation spectrum of laser emission was studied. The inset sketches the experimental geometry with the stimulated emission emerging from the cleaved facets of the device structure. The pump laser is tuned near the $\mathrm{HH}$ exciton absorption peak in this case, and some of the pump light was intentionally scattered into the spectrometer to track the pump radiation, together with the edge emission. For modest pump excitation ( $\mathrm{e}-\mathrm{h}$ pair density on the order of $10^{11} \mathrm{~cm}^{-2}$ ), stimulated edge emission was obtained at very near the biexciton resonance (from FWM spectra) when the pump was coincident with the exciton absorption peak. More detailed information about the gain spectra and its evolution has been obtained for single ZnSe QWs from time-resolved pump-probe studies [11]. Apart from the measurement of the very large optical gain coefficient, $g>10^{5} \mathrm{~cm}^{-1}$, these experiments also demonstrate the initial pathways for exciton molecular formation by optical pumping at or near the $n=1 \mathrm{HH}$ exciton resonance: (i) direct (i.e. coherent) formation of biexcitons by two photon transitions, and (ii) the generation of a molecular population from the "chemical reaction" of excitons in a dense gas. Henneberger and co-workers have paid special attention to the impact of crystalline compositional disorder on 


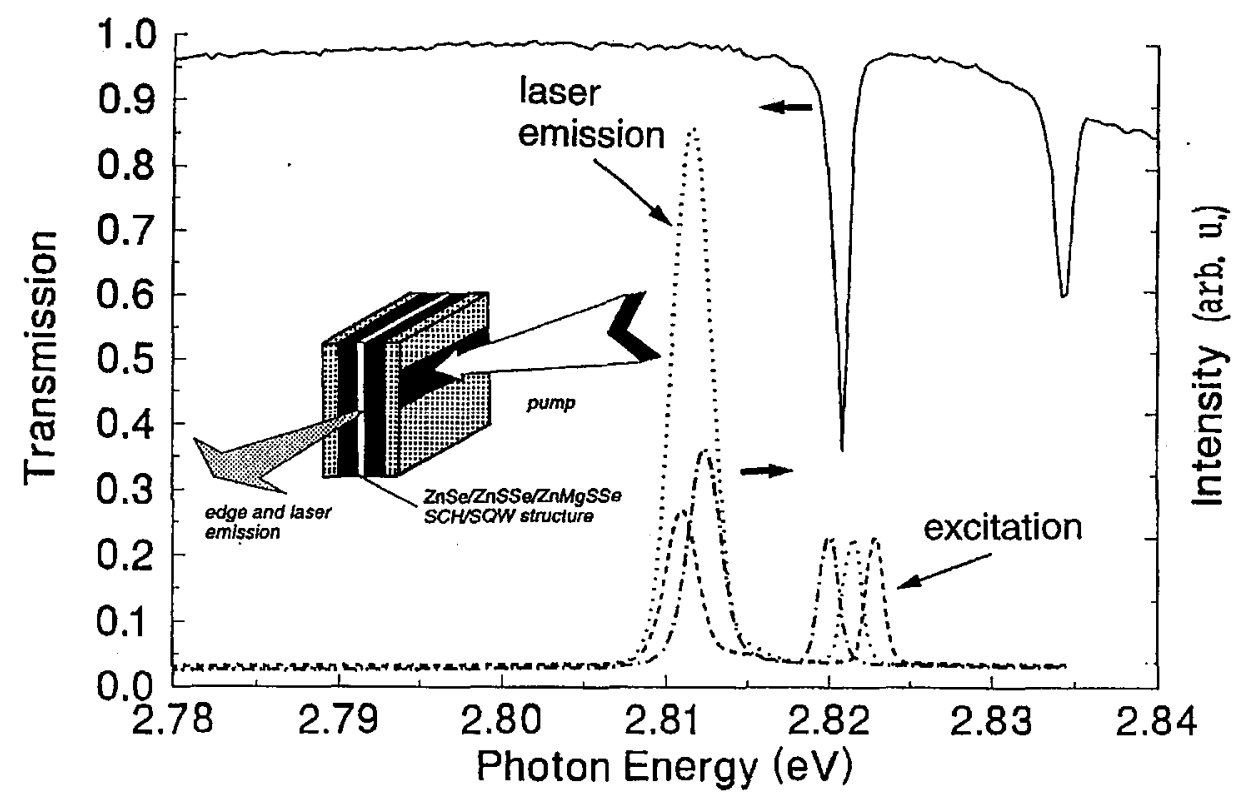

Fig. 5. Bottom trace: excitation spectroscopy of the laser emission via the biexciton transition in a single $75 \AA$ thick $\mathrm{ZnSe} Q W$ at $T=10 \mathrm{~K}$, recorded by ps pump pulses near the $\mathrm{HH}$ exciton resonance. The inset shows the experimental schematic. Top trace: transmission spectrum showing the intense $\mathrm{HH}$ and $\mathrm{LH}$ exciton absorption in a single pass transmission experiment (after Ref. [7]).

the biexciton gain and dynamics [12]. These authors show how the molecular binding energy increases up to $10 \mathrm{meV}$ by confinement in the random alloy potential fluctuations and that biexcitonic gain in an $\mathrm{ZnCdSe} \mathrm{QW}$ may dominate over other processes up to temperatures as high as $T=200 \mathrm{~K}[13]$.

\section{Optical gain in wide gap diode lasers at room temperature}

Of both basic and applied importance is the question as to the microscopic properties of optical gain in a room temperature electroluminescent, i.e. $p n$-junction laser device. As already implied in the introduction, the gain in both ZnSe- and GaN-based QWs diode lasers can be expected to be impacted by a strong excitonic enhancement based on theoretical considerations $[14,15]$. Theory, powered by the so-called semiconductor Bloch equation formalism, is somewhat handicapped in wide gap semiconductors, however, in that the fraction of excitons (to free carriers) in a low threshold room temperature device may be substantial enough so as to introduce an uncertainty to the treatment of screening in the many-body calculation. Experimental input is thus essential, particularly in the form of actual gain spectroscopy.

Figure 6 shows a comparison between gain spectra of a $\mathrm{ZnCdSe} \mathrm{QW}$ green/blue diode laser [16] and a blue InGaN QW diode laser [17], as a function of electrical injection (current) at room temperature. The spectra were obtained 

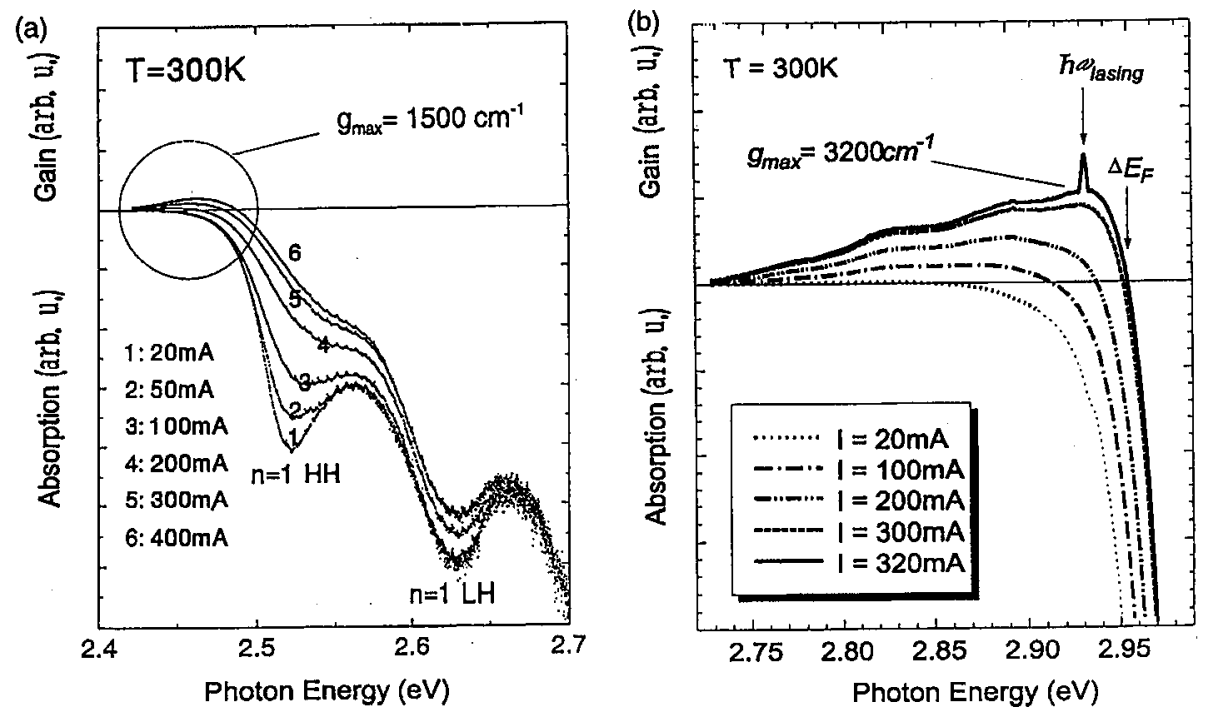

Fig. 6. Comparison between the gain/absorption spectra of a $\mathrm{ZnCdSe} Q W(a)$ and In GaN QW (b) diode laser at room temperature, as a function of injection current. The $n=1 \mathrm{HH}$ and $\mathrm{LH}$ exciton transitions are indicated in the left panel (where the vertical amplitude factor is compressed to display also the exciton absorption features) (after Refs. [16] and [17]).

in each case by the same experimental technique of correlating edge stimulated emission with top spontaneous emission [18]. The energy range in both figures is comparable; however, the details of the actual gain region for the $\mathrm{ZnCdSe}$ is rather narrow (circled region), approximately $30 \mathrm{meV}$, and is enlarged in Fig. 7 for further detail [19]. In striking contrast, the gain for the InGaN laser is spread over an energy range of nearly $200 \mathrm{meV}$. Since the gain spectra for the II-VI laser shows important detail which is masked by compositional and microstructural disorder in the InGaN device, we will first consider the former in terms of the physical message in the left panel of Fig. 6, and comment on the nitride laser shortly below.

When comparing the gain spectrum of the $\mathrm{ZnCdSe} \mathrm{QW}$ laser with that of a high grade GaAs QW device [20], not shown here, one first notes how, as a function of increasing current injection, the $\mathrm{HH}$ and $\mathrm{LH}$ exciton features are rapidly bleached in the GaAs QW so that at laser threshold any semblance to excitonic resonances is absent. This is in striking contrast with the case for the II-VI laser, where a partially bleached $\mathrm{HH}$ exciton resonance and a nearly intact $\mathrm{LH}$ resonance are clearly present at laser threshold, with gain appearing some $60 \mathrm{meV}$ below the $\mathrm{HH}$ exciton resonance. While in broad agreement with predictions of the semiconductor Bloch equations [14, 15], important differences remain concerning the details of the gain spectrum. In the author's opinion, the microscopic details of the system, in terms of the appropriate many-body description remain theoretically incomplete for now. The role of the Coulomb interactions remains practically 


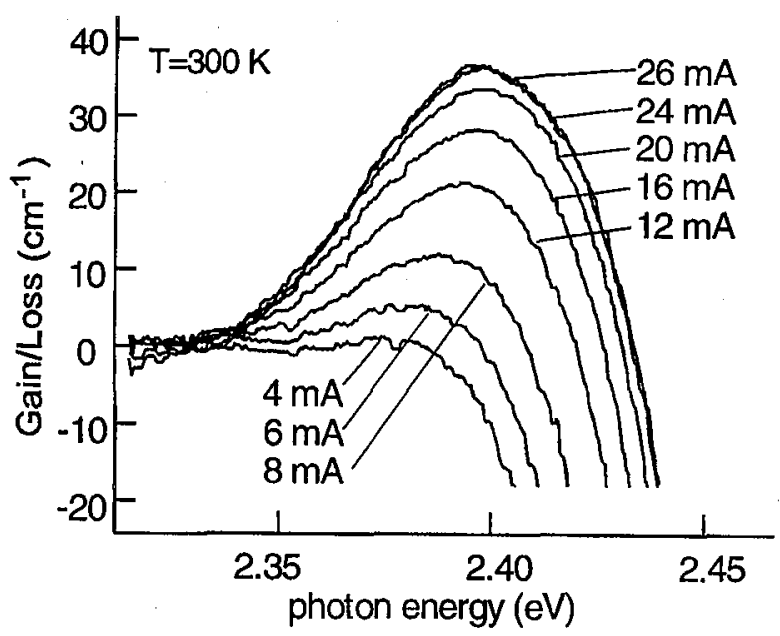

Fig. 7. Details of the gain spectrum for a room temperature ZnCdSe SQW diode laser (an index guided device) (after Ref. [19]).

relevant, for example, in the context of present efforts to fabricate wide band gap semiconductor quantum wire lasers where the bound exciton states might be expected to be increasingly stable against dissociation and screening. In any event, the typical range of enhancement of the peak gain for room temperature $\mathrm{ZnCdSe}$ QW lasers is in the range of factor of three to four, clearly an important positive feature in designing low threshold devices. In considering the laser threshold condition, we remark that while the excitonic enhancements also imply an accelerated spontaneous (radiative) decay rate, the concentration of oscillator strength in the gain spectrum near the $n=1 \mathrm{HH}$ exciton resonance specifically enhances the peak gain coefficient, whereas the spontaneous emission rate is proportional to the integral of the corresponding spontaneous emission spectrum. In particular, with new exploratory II-VI and group III-nitride lasers, where nonradiative recombination may be appreciable, the excitonic enhancements to both spontaneous emission and optical gain are clearly important.

As yet another experimental illustration of the impact of the e-h Coulomb correlation at room temperature, we show in Fig. 8 recent results where $\mathrm{ZnCdSe}$ QW diode lasers have been studied in a magnetic field [21] (bottom trace), while being compared with GaAs QW diode lasers (field applied perpendicular to the QW layer plane) [22]. In simplest terms, the experiment gauges the excitonic strength of the e-h pair system against the predictable Landau quantization of the conduction and valence band edge states in a free electron-hole plasma (in one-electron approximation). In their study of optically pumped $\mathrm{ZnCdSe}$ lasers at cryogenic temperatures, Cingolani and co-workers earlier showed that, with sufficiently large electronic confinement (typically provided in QWs with the Cd-concentration $x>0.20$ ), the quasi-2D system remains excitonic while supplying gain near the $n=1 \mathrm{HH}$ resonance [23]. In these experiments, evidence for a Landau-type shift, dominated by the free electron contribution the confinement was relatively 


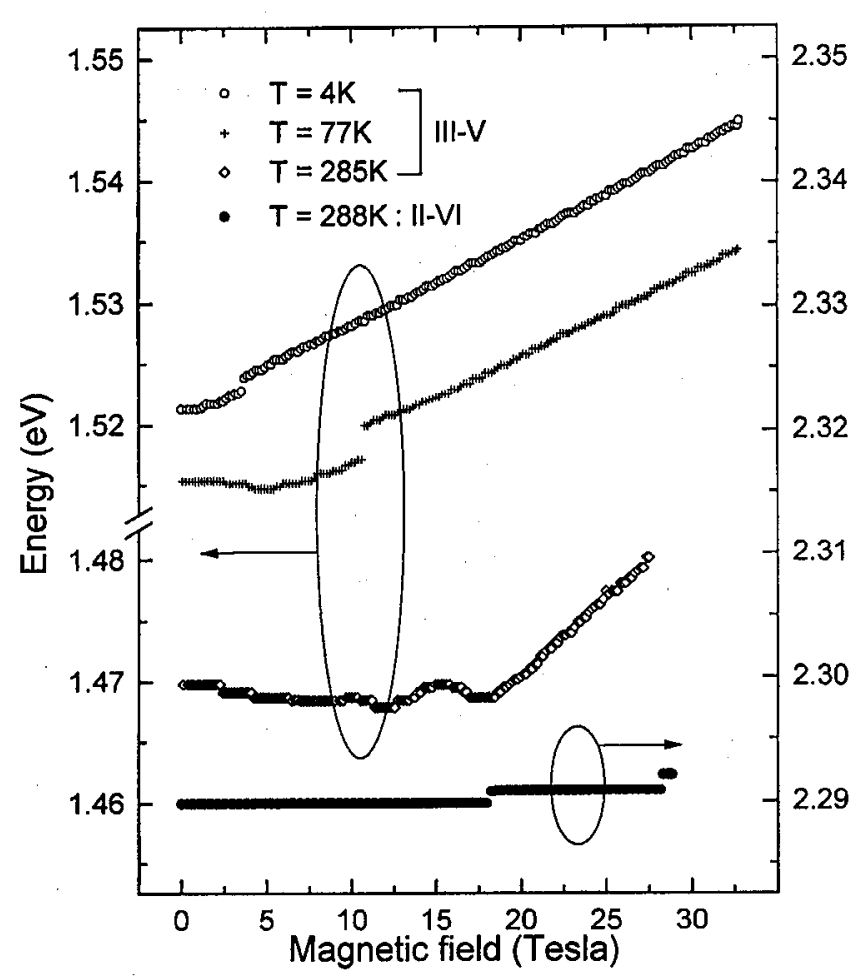

Fig. 8. The emission photon energy for a GaAs QW diode laser (upper traces) and ZnCdSe QW diode laser (lowest trace) as a function of magnetic field, oriented perpendicular to the layer plane.

weak $(x \approx 0.11)$. The shifts in the emission spectrum of the $\mathrm{ZnCdSe} \mathrm{QW}$ index guided laser operating in a single longitudinal mode shown up in Fig. 8 as discrete mode hopping jumps between the photon energies of $h \omega=2.2899 \mathrm{eV}$ (for $B=0-18 \mathrm{~T}$ ), $h \omega=2.2901 \mathrm{eV}(B=18-28 \mathrm{~T}$ ), and $h \omega=2.2921 \mathrm{eV}$ $(B=28-29 \mathrm{~T})$. The diode laser was operated well above threshold at injection level of $I=18 \mathrm{~mA}\left(\approx 1.4 I_{\mathrm{th}}\right.$, where $\left.I_{\mathrm{th}}=13 \mathrm{~mA}\right)$. From the measured room temperature electron-hole lifetime under comparable injection [16], one obtains an e-h pair density of $1.9 \times 10^{12} \mathrm{~cm}^{-2}$ in the ZnCdSe single QW. In a free carrier picture, a Landau shift, linear in the magnetic field and of the order of $\Delta E_{\mathrm{L}} \approx 0.5 \mathrm{meV} / \mathrm{T}$ would be expected (either due to the shift of the lowest conduction band Landau level or the jumps of the Fermi level between such occupied levels). Clearly, the experimental result in Fig. 8 is about one order of magnitude smaller and incompatible with free electron-hole behavior. On the other hand, the agreement is much better when compared with the diamagnetic shifts measured for the $n=1$ $\mathrm{HH}$ exciton transition in ZnCdSe QWs, typically, $\Delta E_{\mathrm{dia}} \approx 2 \mu \mathrm{eV} / \mathrm{T}^{2}$, with the reduced exciton mass of $\mu^{*}=0.1 m_{0}$. Given the exciton binding energy of about $40 \mathrm{meV}$, this implies that at $B=30 \mathrm{~T}$, we are still in the regime where $E_{x}>h \omega_{c}$, the electron cyclotron energy. In strong contrast, while exhibiting temperature de- 
pendent "Landau-level jumping" (oscillations) of the Fermi level at low $B$-fields (in terms of the Fermi-level), the spectral shifts in the emission of the GaAs QW diode laser in Fig. 8 evolve into a linear cyclotron-like shift, with some influence of the many-body interactions on the effective masses evident, however.

We close this section by returning to the gain spectra of the InGaN/GaN diode laser QW system of Fig. 6, which forms the active medium of the spectacularly recently successful blue/violet diode lasers [24]. Even the best of these lasers show threshold current densities which are large, well above $1 \mathrm{kA} / \mathrm{cm}^{2}$. Several recent spectroscopic investigations addressing the linear optical properties of the InGaN QWs at the band edge have shown that this ternary departs appreciably from a random alloy. This can be qualitatively expected by noting the large differences in the covalent component of the bond energies for the two binary end points, respectively, leading to a degree of "immiscibility". Nakamura and co-workers have gone as far as arguing for the presence of "quantum dots" in the active medium [25], while the others have simply pointed out the significant degree of electronic localization that occurs near the band edges [26]. In any event, the absorption edge of the InGaN QW is characterized by a very long, disorder broadened density of states tail (in excess of $100 \mathrm{meV}$ ) from which the low injection electroluminescence (basis of the blue LED) originates. As one consequence of the impact of the microstructural disorder, the absorption edge of InGaN QWs (as studied e.g. by photocurrent spectroscopy [27]) is void of the kinds of distinct features that would identify the $n=1 \mathrm{QW}$ confined particle transition (with associated excitonic enhancements) in e.g. a $\mathrm{ZnCdSe} \mathrm{QW}$.

A key result in Fig. 6 is the large linewidth and the spectral location of the gain relative to the InGaN QW joint density of states as obtained from the absorption spectrum. In the InGaN QW devices, the roughly extrapolated gain band width is in excess of $100 \mathrm{meV}$. This is comparable to the broad electroluminescence (EL) spectrum which is typical also of the commercial blue SQW LED devices. With increasing current, the gain emerges within the low energy tail of the "soft" absorption edge of the InGaN QW with a maximum slightly redshifted with respect to the EL spectrum. These states must be filled first, before the QW can generate useful optical gain. Assuming that the measured net gain is not significantly influenced by extrinsic optical losses (scattering etc.), we note that when compared with the $\mathrm{ZnCdSe} \mathrm{QW}$ diode laser [16], the gain coefficient of the InGaN QW per unit $e-h$ pair density is about one order of magnitude smaller.

It is well known that epitaxially grown GaN-based heterostructures, particularly those on sapphire substrates, display a complex crystalline microstructure, influenced by extrinsic and intrinsic defects. Many different spatial scales are involved, including disorder from extended defects (e.g. dislocations) on the micrometer scale. The optical probes used in our experiments are most sensitive for disorder in an opposite limit, i.e. atomic scale fluctuations in the crystalline potential that affect the band edge states. The microscopic details of the atomic scale disorder in the InGaN/GaN QW are not known at this time but most likely include a combination of compositional and topological fluctuations in the QW layer plane. One extreme limit of the fluctuations is an assembly of InGaN (or InN) clusters, within which the electronic states are defined by a local electronic 
structure. Topological continuity then depends on the proximity of the system to the percolation limit (i.e. extended states associated with interconnected clusters). Of course, the configurational details of the electron and hole confinement impact their wave function overlap and hence the oscillator strength. An interesting contemporary development is the development of lateral epitaxial overgrowth which has dramatically reduced the dislocation density in InGaN heterostructures [28]. It remains to be seen, whether and how the elimination of extended defects has any impact on the indium compositional anomalies which are so strongly evident in the data of Fig. 6.

\section{Vertical microcavity lasers}

In this section we focus on recent research on wide gap semiconductor optical microcavities, where one goal of current research is to elucidate the physics of optical gain and stimulated emission in blue/green vertical cavity emitters within a coupled electron-electromagnetic system. Physics of microcavities in GaAs-based QWs has been subject of significant interest in the past three, four years and much understanding has been obtained especially for the linear optical properties of the exciton-polariton normal modes in these III-V systems [29]. Contrasting with atomic systems, where much pioneering work in the area of light-matter interactions in very high $Q$-factor optical resonators has been witnessed, the situation in the case of highly excited semiconductors differs in a fundamental way. First, the electronic states (e.g. excitons) are composed from spatially extended (Bloch) states. Second, in the optically very dense semiconductor medium, the exciton-exciton interactions must be viewed from the context of a gas of interacting fermions, as modified by the "photon dressing" in a microresonator. While we will not review recent work on highly excited III-V semiconductor microcavity systems here, except to note important recent results from Wang and co-workers [30] and Gibbs and co-workers [31]. Rather, our aim is to offer some insight to the still unfolding saga of the issues associated with optical gain and excitonic interactions within wide gap semiconductor microcavities. Since the development of the III-nitride materials has not at this writing advanced sufficiently for such studies, we will again use the recent work on ZnSe-based QW heterostructures as the primary example. The wide gap semiconductors provide an unusual opportunity for microcavity studies, given their large excitonic oscillator strength. As a basic measure of the strength of exciton-photon interaction in the linear optical regime, normal mode (Rabi) splittings of about $\Omega \approx 10 \mathrm{meV}$ per $\mathrm{QW}$ have been recently measured in a $\mathrm{ZnCdSe} \mathrm{QW}$ microcavity [32] and sizable effects have also been seen in CdTe microcavities [33, 34]. As cliscussed in the preceding sections, the strong electron-hole Coulomb correlations given the measured threshold carrier density in an optically pumped vertical cavity II-VI surface emitting laser [35] and the known optical characteristics of a ZnSe-based QWs at comparable densities, a II-VI microcavity can be expected to reach the lasing threshold before the composite oscillators is saturated by phase-space filling and screening of the excitonic resonance. Then, important basic questions can be addressed in the laboratory about the manifestation of optical gain for the exciton-photon oscillator in the so-called strong coupling (beyond perturbation) regime. 
In the author's laboratory optically pumped short vertical cavity surface emitting laser operation has been obtained in $\mathrm{ZnCdSe} \mathrm{QW}$ microresonator structures up to room temperature. Currently there are several early efforts to try to fabricate also blue nitride based vertical cavity structures - a considerable technical challenge. The actual gain/absorption spectra are shown in Fig. 9 for several different injection levels, ranging from near threshold $\left(P_{\mathrm{th}} \approx 2 \times 10^{12} \mathrm{e}-\mathrm{h}\right.$ pairs per $\left.\mathrm{cm}^{2}\right)$ to low excitation ( $2 \%$ of $P_{\text {th }}$ ) [36]. The characteristics of the gain/absorption spectrum near the threshold demonstrate clearly that the composite exciton-photon oscillator is not completely bleached even at $P_{\text {th }}$. The gain first starts to appear on the low energy side of the cavity-like resonance when the excitation condition reaches about $70 \%$ of the lasing threshold. The maximum gain at threshold is calculated to be 0.0025 . This value agrees well with the mirror reflectivity, anticipated scattering losses etc. and corresponds to one pass gain coefficient of $1500 \mathrm{~cm}^{-1}$, accounting for the two effective QWs.

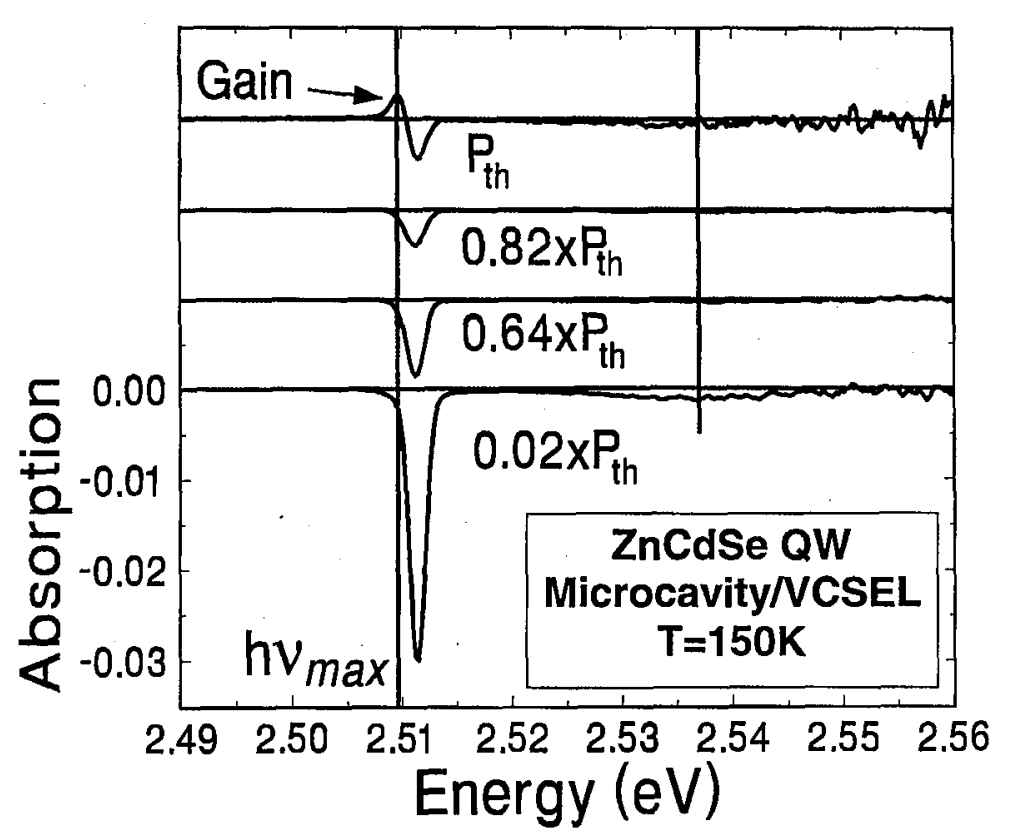

Fig. 9. Gain/absorption spectra for a $\mathrm{ZnCdSe} \mathrm{QW}$ short length $((5 / 2) \lambda)$ vertical cavity surface emitting laser at $T=150 \mathrm{~K}$, as a function of excitation power, relative to the threshold $\left(P_{\mathrm{th}}\right)$ (after Ref. [36]).

We wish to emphasize how the spectroscopic result in Fig. 9 shows the simultaneous existence of gain and absorption in a remarkably narrow spectral range near the threshold. The width of the gain spectrum is only about $2 \mathrm{meV}$ even at $150 \mathrm{~K}$. By contrast, the gain band width for the $\mathrm{ZnCdSe} \mathrm{QW}$ edge emitting lasers is broadened up to $30 \mathrm{meV}$ for this temperature range [16] through exciton-LO phonon scattering. Such broadening would also be typical of a GaAs QW edge 
emitting device. In the case of the II-VI microcavity laser, the anomalous spectral shape of the gain can be attributed to the initial sharpness of the cavity-like polariton branch which in turn acquires its narrow linewidth from the selective enhancement of certain electromagnetic modes by the resonator. The simultaneous existence of the gain and absorption in narrow spectral range and pinning of chemical potential at the low energy side of the cavity mode suggests a large density of states of the combined exciton-photon oscillator in the cavity-like mode. This behavior illustrates emphatically how in a wide gap semiconductor microresonator laser the strong interaction between the QW excitons and the electromagnetic modes transcends the conventional perturbative regime. Other experimental evidence, which further elucidates the transformed nature of the excitons in a microcavity, especially with respect to the large density of the states available in the cavity-like mode, has been obtained from time resolved photoluminescence experiments on the vertical cavity laser structures, as described in [36]. A central result in such studies of vertical cavity II-VI lasers has been the demonstration that, unlike in GaAs-based microresonators, the exciton-cavity normal mode splitting in a ZnSe-based QW system does not vanish at high e-h pair densities. In fact, even under excitation conditions that reach into the vertical cavity lasing regime, the composite exciton-photon oscillator is not saturated. This provides a unique opportunity for studying gain and stimulated emission in a semiconductor optical structure in the regime where the light-matter interaction cannot be treated perturbatively.

\section{Concluding remarks}

In this overview, an attempt has been made to summarize those special physical features which give wide band gap semiconductors such special attributes in their stimulated emission at blue and green wavelengths. These properties, dominated by the exceptionally strong electron-hole Coulomb interactions, offer a fascinating landscape for the optical physicist and set the nitride and II-VI based quantum well heterostructures apart from other contemporary semiconductor nanostructures. The excitonic enhancements are also of applied importance in the design of future high efficiency diode lasers for a wide range of technological applications.

The author is grateful to many students and colleagues at Brown University and elsewhere, whose contributions are reflected in the reference list. I wish especially acknowledge Professor R.L. Gunshor at Purdue University for a unique collaboration.

\section{References}

[1] M. Haase, J. Qiu, J. DePuydt, H. Cheng, Appl. Phys. Lett. 59, 1272 (1991); H. Jeon, J. Ding, W. Patterson, A.V. Nurmikko, W. Xie, D. Grillo, M. Kobayashi, R.L. Gunshor, ibid. 59, 3619 (1991).

[2] S. Nakamura, G. Fasol, The Blue Laser Diode, Springer, Berlin 1997.

[3] N.T. Pelekanos, J. Ding, M. Hagerott, A.V. Nurmikko, H. Luo, N. Samarth, J. Furdyna, Phys. Rev. B 45, 6037 (1992). 
[4] H. Amano, in: Gallium Nitride, in series Semiconductors and Semimetals, Vol. 50, Eds. J. Pankove, T. Moustakas, Academic Press, New York 1998, chapter by B. Monemar, p. 313.

[5] M. Hagerott, H. Jeon, J. Ding, A.V. Nurmikko, W. Xie, D.C. Grillo, M. Kobayashi, R.L. Gunshor, Appl. Phys. Lett. 60, 2825 (1992).

[6] T. Hauptl, H. Nickolaus, F. Henneberger, A. Schulzgen, Phys. Status Solidi B 194, 219 (1996).

[7] V. Kozlov, P. Kelkar, A.V. Nurmikko, C.-C. Chu, D.C. Grillo, J. Han, C.G. Hua, R.L. Gunshor, Phys. Rev. B 53, 10837 (1996).

[8] H. Zhou, A.V. Nurmikko, C. Chu, J. Han, R. Gunshor, T. Takagahara, Phys, Rev. $B$ 58, 10131 (1998).

[9] W. Schäfer, D.S. Kim, J. Shah, T.C. Damen, J.E. Cunningham, K. Gossen, L.N. Pfeiffer, K. Köhler, Phys. Rev. B 53, 16429 (1996).

[10] F. Kreller, M. Lowisch, J. Puls, F. Henneberger, Phys. Rev. Lett. 75, 2420 (1995).

[11] V. Kozlov, P. Kelkar, A.V. Nurmikko, C.-C. Chu, D.C. Grillo, J. Han, C.G. Hua, R.L. Gunshor, Phys. Rev. B 54, 13932 (1996).

[12] J. Puls, H.-J. Wunsche, F. Henneberger, Chem. Phys. 210, 235 (1996).

[13] F. Keller, J. Puls, F. Henneberger, Appl. Phys. Lett. 69, 2406 (1996).

[14]. C. Ell, H. Haug, J. Opt. Soc. Am. B 6, 2006 (1989) and private communication for $\mathrm{ZnSe}$.

[15] W.W. Chow, S.W. Koch, Appl. Phys. Lett. 66, 3004 (1995).

[16] J. Ding, M. Hagerott, P. Kelkar, A.V. Nurmikko, D.C. Grillo, Li He, J. Han, R.L. Gunshor, Phys. Rev. B 50, 5787 (1994).

[17] Y.-K. Song, M. Kuball, A.V. Nurmikko, G.E. Bulman, K. Doverspike, S.T. Sheppard, T.W. Weeks, M. Leopard, H.S. Kong, H. Dieringer, J. Edmond, Appl. Phys. Lett. 72, 1418 (1998).

[18] C.H. Henry, R.A. Logan, F.R. Merritt, J. Appl. Phys. 51, 3042 (1980); P. Blood, A. Kucharska, J. Jacobs, K. Griffiths, J. Appl. Phys. 70, 1144 (1991).

[19] V. Kozlov, A. Salokatve, A.V. Nurmikko, D. Grillo, L. He, J. Han, Y. Fan, M. Ringle, R. Gunshor, Appl. Phys. Lett. 65, 1863 (1994).

[20] M.P. Kresler, C. Harder, Appl. Phys. Lett. 57, 123 (1990).

[21] Y.-K. Song, A.V. Nurmikko, C.-C. Chu, T.B. Ng, R.L. Gunshor, Appl. Phys. Lett. 71, 2874 (1997).

[22] Y.-K. Song, A.V. Nurmikko, unpublished.

[23] R. Cingolani, L. Calcanile, G. Coli, R. Rinaldi, M. Lomoscolo, M. DiDio, A. Franciosi, L. Vanzetti, G.C. LaRocca, D. Campi, J. Opt. Soc. Am. B 13, 1268 (1996).

[24] S. Nakamura, M. Senoh, S. Nagahama, N. Iwasa, T. Yamada, T. Matsushita, H. Kiyoku, Y. Sugimoto, T. Kozaki, H. Umemoto, M. Sano, K. Chocho, Appl. Phys. Lett. 72, 211 (1998).

[25] S. Chichibu, K. Wada, S. Nakamura, Appl. Phys. Lett. 71, 2346 (1997). 
[26] S. Chichibu, T. Azuhata, T. Sota, S. Nakamura, Appl. Phys. Lett. 70, 2822 (1997); S. Chichibu, T. Azuhata, T. Sota, S. Nakamura, Appl. Phys. Lett. 69, 4188 (1996); E.S. Jeon, V. Kozlov, Y.-K. Song, A. Vertikov, M. Kuball, A.V. Nurmikko, H. Liu, C. Chen, R.S. Kern, C.P. Kuo, M. Craford, Appl. Phys. Lett. 69, 4194 (1996); Y. Narukawa, Y. Kawakami, Sz. Fujita, Sg. Fujita, S. Nakamura, Phys. Rev. $B$ 55, R1938 (1997).

[27] M. Kuball, E.-S. Jeon, Y.-K. Song, A.V. Nurmikko, P. Kozodoy, A. Abare, S. Keller, L.A. Coldren, U.K. Mishra, S.P. DenBaars, D.A. Steigerwald, Appl. Phys. Lett. 70, 2580 (1997).

[28] O.-H. Nam, M. Bremster, T. Zheleva, R.F. Davis, Appl. Phys. Lett. 71, 2368 (1997).

[29] For a topical review on work with semiconductor microcavities see e.g. M.S. Skolnick, T.A. Fisher, D.M. Whittaker, Semicond. Sci. Technol. 13, 645 (1998).

[30] X. Fan, H. Wang, H.Q. Hou, B.E. Hammonds, Phys. Rev. B 56, 15256 (1997).

[31] R. Jin, H.M. Gibbs, G. Khitrova, M.S. Tobin, R.P. Leavitt, D. Boggavarapu, F. Jahnke, S.W. Koch, submitted to Phys. Rev. B.

[32] P. Kelkar, V. Kozlov, A.V. Nurmikko, C.-C. Chu, D.C. Grillo, J. Han, C.G. Hua, R.L. Gunshor, Phys. Rev. B 52, R5491 (1995).

[33] J. Sadowski, H. Mariette, A. Wasiela, R. Andre, Y. Merle d'Aubigné, T. Dietl, Phys. Rev. B 56, 1664 (1997).

[34] F. Quochi, G.R. Hayes, R. Andre, G. Bongiovanni, A. Mura, J.L. Staehli, Le Si Dang, J. Cryst. Growth 184, 754 (1998).

[35] H. Jeon, V. Kozlov, P. Kelkar, A.V. Nurmikko, C.-C. Chu, D.C. Grillo, J. Han, C.G. Hua, R.L. Gunshor, Appl. Phys. Lett. 67, 1668 (1995).

[36] P. Kelkar, V. Kozlov, A.V. Nurmikko, J. Han, C.G. Hua, R.L. Gunshor, Phys. Rev. B 56, 7564 (1997). 